\title{
Fragile topological band in the checkerboard antiferromagnetic monolayer FeSe
}

\author{
Aiyun Luo $\mathbb{D}^{1}$, Zhida Song $\mathbb{D D}^{2}$ and Gang $\mathrm{Xu}^{1 凶}$
}

By means of the first-principles calculations and magnetic topological quantum chemistry, we demonstrate that the low-energy physics in the checkerboard antiferromagnetic (AFM) monolayer FeSe, very close to an AFM topological insulator that hosts robust edge states, can be well captured by a double-degenerate nearly flat band with fragile topology just below the Fermi level. The Wilson loop calculations identify that such fragile topology is protected by the $S_{4 z}$ symmetry, which gives rise to a 2D second-order topological insulator that supports the bound state with fractional charge $e / 2$ at the sample corner. This work provides a platform to study the intriguing properties of magnetic fragile topological electronic states. Previous observations of the edge states and bound states in checkerboard AFM monolayer FeSe can also be well understood in our work.

npj Computational Materials (2022)8:26; https://doi.org/10.1038/s41524-022-00707-9

\section{INTRODUCTION}

Topological matters have attracted extensive interest for their distinct and robust bulk-boundary correspondence ${ }^{1-5}$. In particular, the interplay between the crystal symmetry and electronic bands gives rise to a variety of topological states, such as the topological crystalline insulator ${ }^{6-9}$ and higher-order topological insulator $(\mathrm{TI})^{10-16}$. Moreover, when the magnetism and topological bands are entangled together, it will greatly enrich more exotic topological states ${ }^{17-23}$, such as the quantum anomalous Hall effect $^{20,21}$, axion $\mathrm{Tls}^{24,25}$, and magnetic topological semime$\operatorname{tals}^{26,27}$. More recently, a distinct type of topological state, namely the fragile topology ${ }^{28,29}$, has been proposed. Recent advances in magnetic space groups (MSGs) have also enriched the classification of magnetic fragile topology ${ }^{30,31}$. Different from the stable topology, the fragile topology generally cannot exhibit robust edge states. Meanwhile, it hosts the Wannier obstruction, which means that it should undergo a gap closing to evolve into a trivial insulator. Fragile topology shows many intriguing properties ${ }^{32-37}$. For example, the fragile topologically flat band contributes a nontrivial superfluid weight in 2D superconductor ${ }^{34,35}$, and hence enhances the superconducting transition temperature. Under a varying magnetic field, the corner states of the fragile Hofstadter topological system can pump into bulk by magnetic flux ${ }^{36}$. Besides the conceptual breakthrough, searching for more natural fragile topological materials is highly desirable.

As a wonderful platform to study the emergent phenomena among superconductivity, magnetism, and topological bands, FeSe has generated much attentions recent years, in which high$T_{c}$ superconductivity ${ }^{38-40}$, topological superconductivity ${ }^{41-43}$, topological states ${ }^{44-47}$ have been reported. In particular, Wang et al. have reported that the monolayer FeSe in the checkerboard antiferromagnetic (cb-AFM) phase is a $2 \mathrm{D} \mathrm{AFM} \mathrm{TI}{ }^{46}$. After that, edge states are observed at the nematic domain boundaries of monolayer $\mathrm{FeSe}^{47}$. A bound state near the Fermi level is further observed at the end of boundaries. However, the topological origin of the bound state has not been discussed, and the topological nature in the $\mathrm{cb}-\mathrm{AFM}$ monolayer FeSe remains elusive until now.
In this work, by using the first-principles calculations and magnetic topological quantum chemistry ${ }^{2,23,48-51}$, we systematically study the topological properties of the cb-AFM monolayer FeSe. We demonstrate that their low-energy physics can be well captured by a double-degenerate nearly flat band with fragile topology just below the Fermi level, which gives rise to a 2D second-order topological insulator (SOTI) that supports the bound state with fractional charge $e / 2$ at the sample corner. The winding of square Wilson loop confirms that such fragile topology is protected by the $S_{4 z}$ symmetry, which may lead to intriguing response to disorder and magnetic field. Further analyses find that cb-AFM monolayer FeSe is very close to a well-defined AFM TI with nontrivial spin-Chern number characterized by the $S_{z}$ symmetry. These results can well explain the previous observations of topological edge states and bound state near the Fermi level ${ }^{46,47}$, and also provide a platform to study the intriguing properties of the magnetic fragile topology.

\section{RESULTS}

\section{Crystal and magnetic structure}

As illustrated in Fig. 1a, b, both bulk and monolayer FeSe adopt the tetragonal lattice structure with the inversion symmetric space group P4/nmm (No. 129), in which Fe atoms occupy the Wyckoff position $2 a(0,0.5,0)$, and Se atoms occupy the Wyckoff position $2 c^{\prime}(0,0, z)$. In this work, we mainly focus on the band structures and topological properties in the cb-AFM phase of monolayer FeSe as shown in Fig. $1 \mathrm{a}, \mathrm{c}$, d. In this magnetic configuration, the nearest neighboring Fe ions are antiferromagnetically coupled to each other and the inversion symmetry is broken, leading to a MSG $P 4^{\prime} / n^{\prime} m^{\prime} m$ (No. $129.416^{52}$ ). $P 4^{\prime} / n^{\prime} m^{\prime} m$ is generated from a space-time inversion $\mathcal{P} \mathcal{T}$, a twofold screw $C_{2 x}\left(\left\{\hat{C}_{2 x} \mid \frac{1}{2}, 0,0\right\}\right)$, and an improper fourfold rotation $S_{4 z}\left(\left\{\hat{C}_{4 z} \hat{M}_{z} \mid 0, \frac{1}{2}, 0\right\}\right)$.

\section{Electronic structures and fragile topology}

First, we carry out the calculations without spin-orbit coupling (SOC) of the cb-AFM monolayer FeSe, and plot the corresponding projected density of states (DOS) of the $3 d$-orbitals on $\mathrm{Fe}_{2}$ ion 
(a)

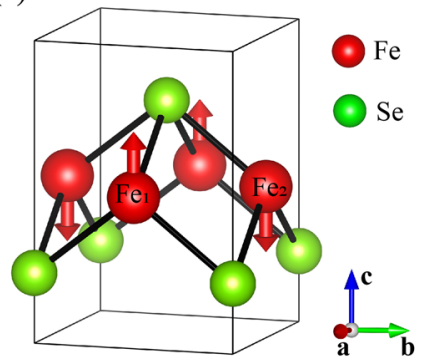

(c)

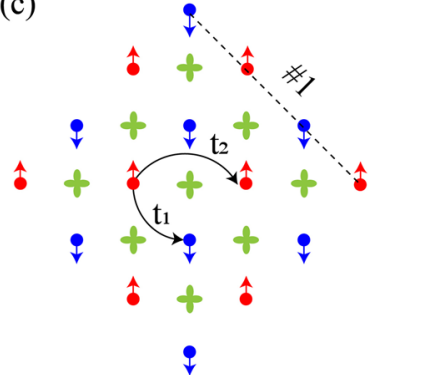

(b)

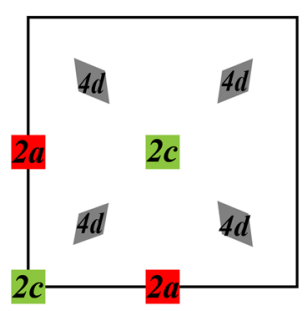

(d)

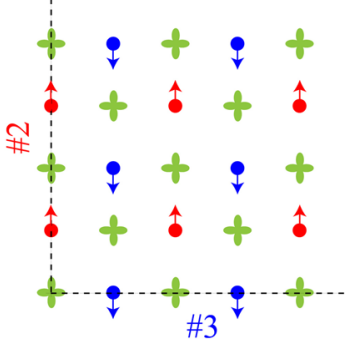

Fig. 1 Illustration of the unit cell and three boundary cuts for cbAFM monolayer FeSe. a The crystal and magnetic structure of monolayer FeSe. b Maximal symmetry Wyckoff positions in MSG P4'/ $n^{\prime} m^{\prime} m$. Since monolayer FeSe is 2D, only the $z=0$ Wyckoff positions are manifested. c, $\mathbf{d}$ Three distinct boundary cuts. Cut \#1 represents the boundary of AFM edge. Cuts \#2 and \#3 represent the boundary of FM edge with spin-up and spin-down polarization along c-direction.


Fig. 2 The electronic structures of cb-AFM monolayer FeSe. a Projected DOS of the $3 d$-orbitals on $\mathrm{Fe}_{2}$ for the calculations without SOC. b Spin-polarized band structures with the red (blue) color indicating the minority (majority) spin states. $c$ Band structures with SOC, the red and blue color indicate the weights of $d_{z^{2}}$-orbital and $d_{y z / z x}$-orbital, respectively. d The Wilson loop spectra of the isolated nearly flat band. The red (blue) lines represent the square (rectangle) Wilson loop spectra with loops patch in the upper (lower) right inset panel, where the arrow indicate the direction of loops.

(see Fig. 1a) in Fig. 2a. It gives rise to a Zeeman splitting about of $2.5 \mathrm{eV}$ between the majority spin states and minority spin states. As a result, most majority spin states are pushed below $-0.25 \mathrm{eV}$, while the states between $-0.25 \sim 2.25 \mathrm{eV}$ are mainly contributed by the minority spin $3 d$-orbitals. Further analysis demonstrates that the

low-energy bands around the Fermi level $(0 \mathrm{eV})$ are dominated by the minority spin of $d_{z^{2}}$ and $d_{y z / x z}$-orbital, which have very weak hybridization with the majority spin components as shown in Fig. 2a. These features can also be represented by the spin-polarized band structures as shown in Fig. 2b, which indicates that five majority spin bands are fully occupied and only one minority band is occupied. Such occupancy actually coincide with the $3 d^{6}$ configuration of the $\mathrm{Fe}^{2+}$ ion. Further detailed orbital components analysis demonstrates that the one occupied minority band is mainly contributed by the $d_{z^{2}}$-orbital, except a band inversion with the $d_{y z / x z}$-orbital at the $M$ point near the Fermi level (see Fig. 2c). The band inversion leads to a semimetallic feature with a parabolic touching at the $M$ point, as shown in Fig. 2b. When the SOC is included, the gapless touching of $d_{y z}$ and $d_{x z}$-orbital at $M$ point is opened with an insulating gap about of $50 \mathrm{meV}$. Since the dispersion of the $d_{z^{2}}$ band is very weak, the gap opening also leads to an isolated nearly flat band just below the Fermi level, as shown in Fig. 2c. We further note that each band is double-degenerate in the presence of $\mathcal{P} \mathcal{T}$ symmetry.

To investigate the topological properties of the cb-AFM monolayer FeSe, we employ the symmetry analysis under the theory of magnetic topological quantum chemistry $22,23,48-51$, in which the magnetic elementary band representations (MEBRs) as the basis of magnetic atomic insulators (Als) are defined. Within this theory, if the irreducible co-representations (coirreps) of the occupied bands at high symmetry momenta points can be decomposed into the linear combination of MEBRs with positive integer, the system is equivalent to a magnetic Al. Otherwise, the system must be topologically nontrivial. We have calculated the co-irreps of all occupied bands with $\mathrm{SOC}$, which indicate that $\mathrm{cb}-\mathrm{AFM}$ monolayer FeSe is a magnetic $\mathrm{Al}$, but not the AFM TI reported in refs. ${ }^{46,47}$. As pointed out in ref. ${ }^{28}$, the band structures of $\mathrm{Al}$ have two combination of manners: (i) $\mathrm{Al}=\mathrm{Al}+\mathrm{Al}$, (ii) $\mathrm{Al}=\mathrm{Al}+$ fragile topological bands. When we focus on the isolated nearly flat band just below the Fermi level, the calculated co-irreps give rise to $\left\{\bar{\Gamma}_{7}, \bar{X}_{2} \bar{X}_{4}, \bar{M}_{6}\right\}$ as shown in Fig. $2 \mathrm{c}$, and its fragile topological characteristic will be discussed below. Comparing to the MEBRs of the MSG $P 4^{\prime} / n^{\prime} m^{\prime} m$ tabulate in Table 1, one can find that the co-irreps of the isolated nearly flat band can only be expressed as a combination of MEBRs with negative integer:

$\left\{\bar{\Gamma}_{7}, \bar{X}_{2} \bar{X}_{4}, \bar{M}_{6}\right\}=(\bar{E})_{2 c} \uparrow G \ominus\left({ }^{2} \bar{E}_{2}\right)_{2 a} \uparrow G$,

the negative coefficient $\ominus$ manifests that it is a fragile topological band $^{28,29}$.

Such fragile topological characteristic can be usually revealed by the nontrivial winding of Wilson loop along some symmetric path over the whole Brillouin zone $(B Z)^{30,53,54}$. Here, we design a series of square loops $I_{i}$ that satisfy $S_{4 z}$ symmetry, as shown in the upper right of Fig. $2 \mathrm{~d}$, with $l_{0}$ at the $M$ point and $l_{\text {end }}$ corresponding to the boundary of the first BZ. The calculated Wilson loop spectra of the fragile topological band are plotted as red lines in Fig. $2 d$, which give rise to a conjugate pairs $\left\{e^{i \theta}, e^{-i \theta}\right\}$ for each $l_{i}$ yielding to the $\mathcal{P} \mathcal{T}$ symmetry ${ }^{55}$. More importantly, each branch of the Wilson loop spectra evolves a $2 \pi$ phase from the $M$ point to the whole $B Z$, leading to a nontrivial winding number 1 . We also design the other series of rectangle loops that break $S_{4 z}$ symmetry, as shown in the lower right of Fig. 2d. The corresponding Wilson loop spectra are plotted as blue lines in Fig. 2d, which obviously show that the crossing at $\theta= \pm \pi$ is opened, resulting in a trivial winding number 0 . Therefore, we conclude that the fragile topological band in cb-AFM monolayer FeSe is protected by the $S_{4 z}$ symmetry. It may lead to intriguing response to disorder, comparing to the inversion symmetryprotected fragile topological band ${ }^{56,57}$.

In addition to the fragile topology, we would like to point out that the cb-AFM monolayer FeSe is very close to a stable AFM TI. Such stable AFM TI phase is protected by the spin-conserving symmetry $S_{z}$, which decouples the interactions between spin-up 
Table 1. The MEBRs induced by maximal symmetry Wyckoff positions in $P 4^{\prime} / n^{\prime} m^{\prime} m^{22,52}$.

\begin{tabular}{llll}
\hline MEBRs & $\Gamma(0,0)$ & $X(0, \pi)$ & $M(\pi, \pi)$ \\
\hline$\left({ }^{1} \bar{E}_{1}\right)_{2 a} \uparrow G$ & $\bar{\Gamma}_{7}$ & $\bar{X}_{2} \bar{X}_{4}$ & $\bar{M}_{7}$ \\
$\left({ }^{1} E_{2}\right)_{2 a} \uparrow G$ & $\bar{\Gamma}_{6}$ & $\bar{X}_{2} \bar{X}_{4}$ & $\bar{M}_{6}$ \\
$\left({ }^{2} E_{1}\right)_{2 a} \uparrow G$ & $\bar{\Gamma}_{7}$ & $\bar{X}_{3} \bar{X}_{5}$ & $\bar{M}_{6}$ \\
$\left({ }^{2} E_{2}\right)_{2 a} \uparrow G$ & $\bar{\Gamma}_{6}$ & $\bar{X}_{3} \bar{X}_{5}$ & $\bar{M}_{7}$ \\
$(\bar{E})_{2 c} \uparrow G$ & $\bar{\Gamma}_{6} \oplus \bar{\Gamma}_{7}$ & $\bar{X}_{2} \bar{X}_{4} \oplus \bar{X}_{3} \bar{X}_{5}$ & $\bar{M}_{6} \oplus \bar{M}_{7}$ \\
\hline
\end{tabular}

Since the monolayer FeSe is $2 \mathrm{D}$, only the $z=0$ Wyckoff positions and $k_{z}=0$ high symmetry momenta points are considered. The MEBRs are denoted as $D_{w} \uparrow G$, which represent a co-irreps of space group $G$ induced by the $D$ orbital at Wyckoff position $w$.
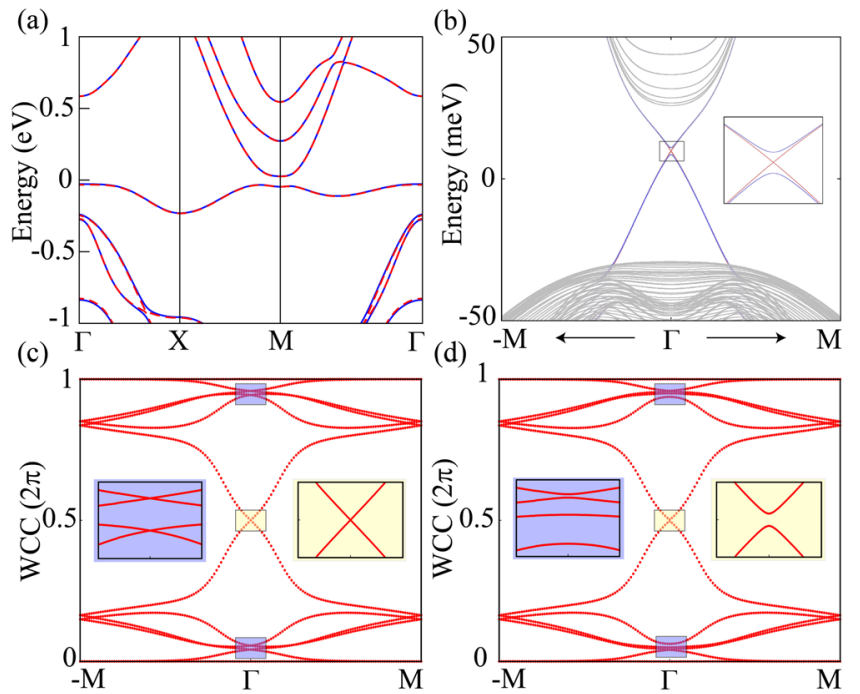

Fig. 3 The electronic structures of Wannier Hamiltonian $H_{0}$ and those with additional $\boldsymbol{S}_{\boldsymbol{z}}$ symmetry $\boldsymbol{H}_{\boldsymbol{s}}$. a Band structures of $\hat{H}_{S}$ (red dash line) and $\hat{H}_{0}$ (blue solid line). b Edge states of the $\hat{H}_{S}$ (red) and $\hat{H}_{0}$ (blue), with a surface onsite energy $-0.1 \mathrm{eV}$. c, d The Wilson loop spectra of all occupied bands in $\hat{H}_{S}$ and $\hat{H}_{0}$, respectively.

and spin-down flavor. In general, the $S_{z}$ symmetry is broken in realistic electron systems ${ }^{58,59}$. However, we demonstrate that the $S_{z}$-breaking interaction in cb-AFM monolayer FeSe is very tiny, which can be verified by two bodies of evidence. First, we have symmetrized the Wannier Hamiltonian $\hat{H}_{0}$ of cb-AFM monolayer FeSe by an additional $S_{z}$ symmetry, i.e. $\hat{H}_{S}=\left(H_{0}+S_{z} \hat{H}_{0} S_{z}^{-1}\right) / 2$, and plot the band structures of $\hat{H}_{S}$ (red dash line) as a comparison to the bands of $\hat{H}_{0}$ (blue solid line) in Fig. 3a. The red bands in Fig. 3a match the blue bands very well, which strongly implies that the $\hat{H}_{0}$ is approximate to having $S_{z}$ symmetry. The approximately $S_{z}$ symmetry is also consistent with our first-principles calculations, which display the weak hybridization between majority spin states and minority spin states as shown in Fig. 2a, b. Second, one can quantitatively estimate the $S_{z}$-breaking process as a competition between the SOC interaction $\lambda_{\mathrm{soc}}$ and the onsite magnetic polarization splitting $\Delta$, where $\lambda_{\text {soc }}$ regards the spin mixing ability and $\Delta$ preserves spin conserving ${ }^{30}$. Reminding that the magnetic splitting $\Delta$ in cb-AFM monolayer FeSe is estimated as $\sim 2.5 \mathrm{eV}$ from our first-principles calculations, and the $\lambda_{\text {soc }}$ of $\mathrm{Fe}$ is usually considered as $\sim 0.03 \mathrm{eV}$. Such a huge energy difference makes the $S_{z}$ symmetry be a good approximation in cb-AFM monolayer FeSe.

When $S_{z}$ symmetry is restored, the system can be considered as two copies of spin-Chern insulators that are connected by the $\mathcal{P} \mathcal{T}$ symmetry. In Fig. 3b, we calculate and plot the edge states of $\hat{H}_{S}$ on the AFM boundary (cut \#1 in Fig. 1c) as red lines, which shows that two edge states cross each other exactly and form a massless Dirac cone at the $\Gamma$ point. As a result, we confirm that the $\hat{H}_{S}$ describes an AFM TI protected by the $S_{z}$ symmetry. As a comparison, we also plot the calculated edge spectra of $\hat{H}_{0}$ on $\# 1$ boundary as the blue lines in Fig. 3b. As expected, the edge spectra of $H_{0}$ are very similar to those of $\hat{H}_{S}$, except a very tiny massive gap about of $0.5 \mathrm{meV}$ at the $\Gamma$ point yielding to the $S_{z}$ symmetry breaking. These results confirm again that the $S_{Z^{-}}$ breaking is very weak in the cb-AFM monolayer FeSe, which is very close to a well-defined AFM TI. This is why the quantum spin Hall-like edge states have been reported and observed in refs. ${ }^{46,47}$. Apart from the edge states, the topological properties can also be revealed by the Wilson loop spectra of all occupied bands ${ }^{60}$. In Fig. $3 c$, d, we plot the Wilson loop spectra along the $\Gamma-M$ path of $\hat{H}_{S}$ and $\hat{H}_{0}$, respectively. The spectra in Fig. $3 c$ obviously show gapless crossing at the time-reversal momenta points, reflecting a well-defined spin-Chern number $\left|C_{s}\right|=1$. However, the Wilson loop spectra of $\hat{H}_{0}$ are generally gaped, indicating that cb-AFM monolayer FeSe is not a well-defined AFM TI.

\section{Corner states}

The fragile topology usually relates to 2D SOTI and leads to filling anomaly ${ }^{15}$. Such filling anomaly could give rise to partially occupied corner states at the sample corner ${ }^{13,15}$. In the system with $S_{4 z}$ symmetry, one can identify 2D SOTI phase by the $S_{4 z}$ eigenvalues of occupied bands ${ }^{12,16}$. We note that for nonsymmorphic MSG $P 4^{\prime} / n^{\prime} m^{\prime} m$, the $S_{4 z}$ eigenvalues at $\Gamma$ point have to come in complex-conjugate pairs $\left\{\xi \exp ^{i \pi / 4}, \xi \exp ^{-i \pi / 4}\right\}$, whilst come in $\left\{\xi \exp ^{i \pi / 4},-\xi \exp ^{-i \pi / 4}\right\}$ at $M$ point and $C_{2 z}$ eigenvalues $\left(C_{2 z}=S_{4 z}^{2}\right)$ at $X$ point come in $\left\{\xi \exp ^{i \pi / 2}, \xi \exp ^{i \pi / 2}\right\}$ with $\xi=+1$ or $\xi=-1$. By counting the numbers of $\xi=+1$ and $\xi=-1$ states at $\Gamma$, $M$ and $\mathrm{X}$ point, we find the following Fu-Kane-like formula could mapping the invariant indicator $Z_{2}$,

$$
(-1)^{Z_{2}}=\prod_{n=1}^{N / 2} \xi_{n}(\Gamma) \xi_{n}(M) \xi_{n}(X),
$$

where $N$ is the number of occupied bands. Comparing with the MEBRs as tabulated in Table 1, we find the MEBRs induced from the atomic site $2 a$ are fall into the $Z_{2}=0$ phase, while $Z_{2}=1$ indicates a $2 \mathrm{D}$ SOTI that would give rise to corner states. The calculated results of all occupied bands are $\xi(\Gamma)=-1, \xi(M)=1$ and $\xi(X)=1$, which certify that cb-AFM monolayer FeSe falls into the 2D SOTI phase with $Z_{2}=1$.

The 2D SOTI property can also be understood from the opposite mass term of the edge states on two different FM boundary (cut $\# 2$ and cut \#3 in Fig. 1d) ${ }^{61}$. We calculate and plot the spinpolarized edge states along the \#2 and \#3 boundary in Fig. $4 a$ and $b$, respectively. In both of which, a massive gap can be observed between two opposite spin-polarized edge states. Further analysis shows that the gap opening on the spin-up boundary (\#2) is induced by a mass term $m_{\# 2} \propto \sigma_{y}$, while the gap opening on the spin-down boundary (\#3) is induced by an opposite mass term $m_{\# 3} \propto-\sigma_{y}$, as shown in the inset of Fig. $4 a$ and $b$, respectively. The opposite mass term is consistent with previous work that two boundaries are related by the $C_{4 z} \mathcal{T}$ symmetry ${ }^{12}$. Therefore, one can expect that the mass terms could cancel each other at the intersection of \#2 and \#3 boundary, which can induce corner states at the $2 c$ position.

In Fig. 4c, we plot the low-energy spectra of a square sample with the center at $2 c$ position ${ }^{31}$ constructed by $20 \times 20$ unit cells by using the the Wannier Hamiltonian $\hat{H}_{0}$. As expected, four corner states including two occupied and two unoccupied (blue dots in Fig. 4c) appear near the Fermi level. However, because $\hat{H}_{0}$ has no chiral symmetry, the four corner states are not pinned at zero 
4

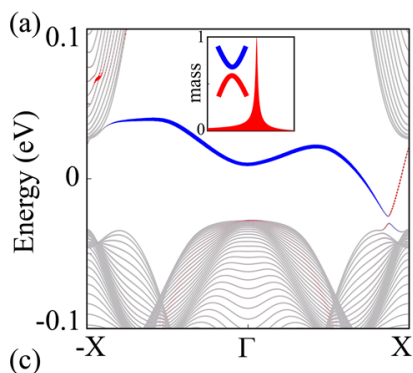

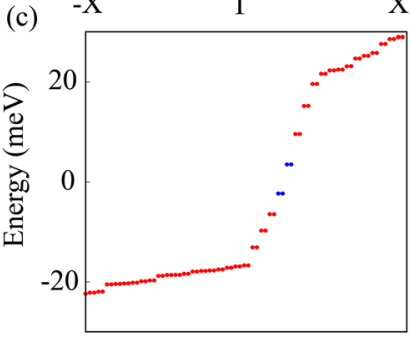

Eigenstate

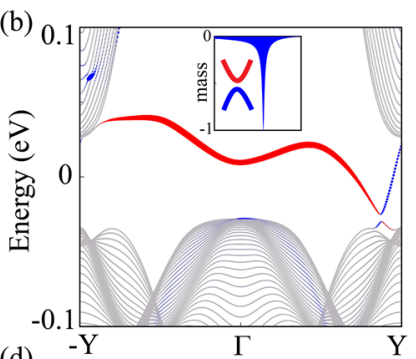

(d)

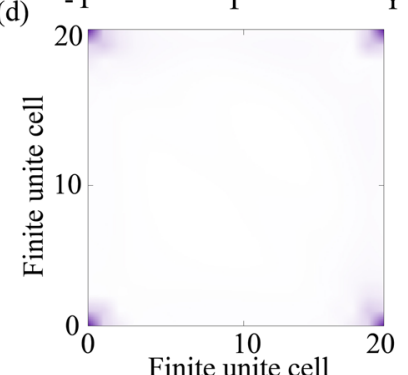

Fig. 4 The edge states and corner states of cb-AFM monolayer FeSe. a, b Spin-polarized edge states along the cuts \#2 and \#3 boundary, respectively. The weights of positive (negative) $\sigma_{z}$ eigenvalue is characterized as red (blue). The inset pictures show the calculated mass term $\sigma_{y}$ near the massive Dirac cone. c Energy spectra of the $20 \times 20$ finite square structure. $\mathbf{d}$ Spatial distribution of the two occupied corner states.

energy exactly. In Fig. 4d, we plot the spatial distribution of the two occupied corner states, which are localized at four corners and each corner has $e / 2$ charge, corresponding to previous conclusions of the 2D SOTI protected by $C_{4 z} \mathcal{T}$ symmetry very well $^{16}$. Finally, we emphasize that such calculated corner states are strongly supported by the previous experimental observations, in which bound state near the Fermi level has been observed at the crossing point of four domain walls by scanning tunneling microscopy ${ }^{47}$. We would like to suggest the experimenters to measure the charge of each bound state, which will be very helpful to confirm its topological origin.

\section{DISCUSSION}

At last, we would like to discuss that the 2D SOTI phase and corner states can only exist in the cb-AFM phase, but cannot be realized in other magnetic phases such as the nonmagnetic or stripe AFM phase. As we all know, the corner states are associated with the mismatch between the atomic sites and the charge centers ${ }^{13}$. As shown in Fig. 1c, d, the electrons on the nearest neighboring Fe ions are oppositely polarized in the cb-AFM configuration, which could strongly suppress the nearest neighbor hopping $t_{1}$ (see Fig. $1 \mathrm{c})$, and makes the next nearest neighbor hopping $t_{2}$ dominate. The dominant $t_{2}$ will lead to the charge centers move to the $2 c$ site, and give rise to corner states at the sample corner. However, $t_{1}$ is always dominant in the NM or stripe AFM configuration. This makes the charge centers locate at the $4 d$ site (see Fig. 1b), which cannot support the corner states appearing at the sample corner. Therefore, our calculations, combining with the observation of the corner states ${ }^{47}$, strongly suggest that the cb-AFM phase is realized in the few layers of the tensile FeSe.

\section{METHODS}

\section{First-principles calculations}

Our first-principles calculations are performed by the Vienna ab initio simulation package ${ }^{62,63}$. Similar as in ref. ${ }^{47}$, Perdew-Burke-Ernzerhof type of the generalized gradient approximation + Hubbard $U(\mathrm{GGA}+U)^{64,65}$ is used as the exchange-correlation potential with $U=1.0 \mathrm{eV}$ and $J=0.2 \mathrm{eV}$ for the Fe $3 d$-orbitals. The crystal parameters $a=3.85 \AA$ and Se atomic coordinates $z=0.255$ are used in all calculations. The cut-off energy for the wave function expansion is set to $400 \mathrm{eV}$, and a $9 \times 9 \times 1 \mathrm{k}$-mesh in the first BZ is used for self-consistent calculations. The SOC is considered selfconsistently. We then construct the Wannier functions without performing maximally localized procedure for the Fe $3 d$-orbitals using WANNIER9066.

\section{DATA AVAILABILITY}

The data that support the findings of this study are available from the corresponding author upon reasonable request.

Received: 16 September 2021; Accepted: 11 January 2022; Published online: 02 February 2022

\section{REFERENCES}

1. Kane, C. L. \& Mele, E. J. Quantum spin hall effect in graphene. Phys. Rev. Lett. 95 , 226801 (2005).

2. Bernevig, B. A., Hughes, T. L. \& Zhang, S.-C. Quantum spin hall effect and topological phase transition in HgTe quantum wells. Science 314, 1757-1761 (2006).

3. König, M. et al. Quantum spin hall insulator state in HgTe quantum wells. Science 318, 766-770 (2007).

4. Zhang, $\mathrm{H}$. et al. Topological insulators in $\mathrm{Bi}_{2} \mathrm{Se}_{3}, \mathrm{Bi}_{2} \mathrm{Te}_{3}$ and $\mathrm{Sb}_{2} \mathrm{Te}_{3}$ with a single Dirac cone on the surface. Nat. Phys. 5, 438-442 (2009).

5. Chen, Y. L. et al. Experimental realization of a three-dimensional topological insulator, $\mathrm{Bi}_{2} \mathrm{Te}_{3}$. Science 325, 178-181 (2009).

6. Fu, L. Topological crystalline insulators. Phys. Rev. Lett. 106, 106802 (2011).

7. Hsieh, T. H. et al. Topological crystalline insulators in the SnTe material class. Nat. Commun. 3, 1-7 (2012).

8. Slager, R.-J., Mesaros, A., Juričić, V. \& Zaanen, J. The space group classification of topological band-insulators. Nat. Phys. 9, 98-102 (2013).

9. Wang, Z., Alexandradinata, A., Cava, R. J. \& Bernevig, B. A. Hourglass fermions. Nature 532, 189-194 (2016).

10. Benalcazar, W. A., Bernevig, B. A. \& Hughes, T. L. Quantized electric multipole insulators. Science 357, 61-66 (2017).

11. Benalcazar, W. A., Bernevig, B. A. \& Hughes, T. L. Electric multipole moments, topological multipole moment pumping, and chiral hinge states in crystalline insulators. Phys. Rev. B 96, 245115 (2017).

12. Schindler, F. et al. Higher-order topological insulators. Sci. Adv. 4, eaat0346 (2018).

13. Song, Z., Fang, Z. \& Fang, C. (d - 2)-dimensional edge states of rotation symmetry protected topological states. Phys. Rev. Lett. 119, 246402 (2017).

14. Xu, Y., Song, Z., Wang, Z., Weng, H. \& Dai, X. Higher-order topology of the axion insulator Euln ${ }_{2} \mathrm{As}_{2}$. Phys. Rev. Lett. 122, 256402 (2019).

15. Wieder, B. J. \& Bernevig, B. A. The axion insulator as a pump of fragile topology. Preprintat https://arxiv.org/abs/1810.02373 (2018).

16. Zou, J., He, Z. \& Xu, G. Higher-order topological insulators in a crisscross antiferromagnetic model. Phys. Rev. B 100, 235137 (2019).

17. Mong, R. S. K., Essin, A. M. \& Moore, J. E. Antiferromagnetic topological insulators. Phys. Rev. B 81, 245209 (2010).

18. Yu, R. et al. Quantized anomalous hall effect in magnetic topological insulators. Science 329, 61-64 (2010).

19. Li, R., Wang, J., Qi, X.-L. \& Zhang, S.-C. Dynamical axion field in topological magnetic insulators. Nat. Phys. 6, 284-288 (2010).

20. Chang, C.-Z. et al. Experimental observation of the quantum anomalous Hall effect in a magnetic topological insulator. Science 340, 167-170 (2013).

21. Deng, Y. et al. Quantum anomalous Hall effect in intrinsic magnetic topological insulator $\mathrm{MnBi}_{2} \mathrm{Te}_{4}$. Science 367, 895-900 (2020).

22. $\mathrm{Xu}, \mathrm{Y}$. et al. High-throughput calculations of magnetic topological materials. Nature 586, 702-707 (2020).

23. Elcoro, L. et al. Magnetic topological quantum chemistry. Nat. Commun. 12, 1-10 (2021).

24. Zhang, D. et al. Topological axion states in the magnetic insulator $\mathrm{MnBi}_{2} \mathrm{Te}_{4}$ with the quantized magnetoelectric effect. Phys. Rev. Lett. 122, 206401 (2019).

25. Liu, C. et al. Robust axion insulator and chern insulator phases in a twodimensional antiferromagnetic topological insulator. Nat. Mater. 19, 522-527 (2020).

26. Xu, G., Weng, H., Wang, Z., Dai, X. \& Fang, Z. Chern semimetal and the quantized anomalous hall effect in $\mathrm{HgCr}_{2} \mathrm{Se}_{4}$. Phys. Rev. Lett. 107, 186806 (2011).

27. Zou, J., He, Z. \& Xu, G. The study of magnetic topological semimetals by first principles calculations. Npj Comput. Mater. 5, 1-19 (2019).

28. Po, H. C., Watanabe, H. \& Vishwanath, A. Fragile topology and Wannier obstructions. Phys. Rev. Lett. 121, 126402 (2018). 
29. Cano, J. et al. Topology of disconnected elementary band representations. Phys. Rev. Lett. 120, 266401 (2018).

30. Bouhon, A., Lange, G. F. \& Slager, R.-J. Topological correspondence between magnetic space group representations and subdimensions. Phys. Rev. B 103, 245127 (2021).

31. Lange, G. F., Bouhon, A. \& Slager, R.-J. Subdimensional topologies, indicators, and higher order boundary effects. Phys. Rev. B 103, 195145 (2021).

32. Song, Z.-D., Elcoro, L. \& Bernevig, B. A. Twisted bulk-boundary correspondence of fragile topology. Science 367, 794-797 (2020).

33. Peri, V. et al. Experimental characterization of fragile topology in an acoustic metamaterial. Science 367, 797-800 (2020)

34. Xie, F., Song, Z., Lian, B. \& Bernevig, B. A. Topology-bounded superfluid weight in twisted bilayer graphene. Phys. Rev. Lett. 124, 167002 (2020).

35. Peri, V., Song, Z.-D., Bernevig, B. A. \& Huber, S. D. Fragile topology and flat-band superconductivity in the strong-coupling regime. Phys. Rev. Lett. 126, 027002 (2021).

36. Herzog-Arbeitman, J., Song, Z.-D., Regnault, N. \& Bernevig, B. A. Hofstadter topology: Noncrystalline topological materials at high flux. Phys. Rev. Lett. 125, 236804 (2020).

37. Lian, B., Xie, F. \& Bernevig, B. A. Landau level of fragile topology. Phys. Rev. B 102, 041402 (2020).

38. Tan, S. et al. Interface-induced superconductivity and strain-dependent spin density waves in $\mathrm{FeSe} / \mathrm{SrTiO}_{3}$ thin films. Nat. Mater. 12, 634-640 (2013).

39. $\mathrm{He}, \mathrm{S}$. et al. Phase diagram and electronic indication of high-temperature superconductivity at $65 \mathrm{~K}$ in single-layer FeSe films. Nat. Mater. 12, 605-610 (2013).

40. Ge, J.-F. et al. Superconductivity above $100 \mathrm{~K}$ in single-layer FeSe films on doped $\mathrm{SrTiO}_{3}$. Nat. Mater. 14, 285-289 (2015).

41. Wang, Z. et al. Topological nature of the $\mathrm{FeSe}_{0.5} \mathrm{Te}_{0.5}$ superconductor. Phys. Rev. $B$ 92, 115119 (2015).

42. Xu, G., Lian, B., Tang, P., Qi, X.-L. \& Zhang, S.-C. Topological superconductivity on the surface of Fe-based superconductors. Phys. Rev. Lett. 117, 047001 (2016).

43. Zhang, P. et al. Observation of topological superconductivity on the surface of an iron-based superconductor. Science 360, 182-186 (2018).

44. Hao, N. \& Hu, J. Topological phases in the single-layer FeSe. Phys. Rev. X 4, 031053 (2014).

45. Zhang, P. et al. Multiple topological states in iron-based superconductors. Nat. Phys. 15, 41-47 (2019).

46. Wang, Z. et al. Topological edge states in a high-temperature superconductor $\mathrm{FeSe} / \mathrm{SrTiO}_{3}$ (001) film. Nat. Mater. 15, 968-973 (2016).

47. Yuan, Y. et al. Edge states at nematic domain walls in FeSe films. Nano Lett. 18, 7176-7180 (2018)

48. Kruthoff, J., de Boer, J., van Wezel, J., Kane, C. L. \& Slager, R.-J. Topological classification of crystalline insulators through band structure combinatorics. Phys. Rev. X 7, 041069 (2017)

49. Po, H. C., Vishwanath, A. \& Watanabe, H. Symmetry-based indicators of band topology in the 230 space groups. Nat. Commun. 8, 1-9 (2017).

50. Bradlyn, B. et al. Topological quantum chemistry. Nature 547, 298-305 (2017).

51. Watanabe, H., Po, H. C. \& Vishwanath, A. Structure and topology of band structures in the 1651 magnetic space groups. Sci. Adv. 4, eaat8685 (2018).

52. Gallego, S. V., Tasci, E. S., Flor, G., Perez-Mato, J. M. \& Aroyo, M. I. Magnetic symmetry in the bilbao crystallographic server: a computer program to provide systematic absences of magnetic neutron diffraction. J. Appl. Crystallogr. 45 1236-1247 (2012).

53. Bouhon, A., Black-Schaffer, A. M. \& Slager, R.-J. Wilson loop approach to fragile topology of split elementary band representations and topological crystalline insulators with time-reversal symmetry. Phys. Rev. B 100, 195135 (2019).

54. Bradlyn, B., Wang, Z., Cano, J. \& Bernevig, B. A. Disconnected elementary band representations, fragile topology, and Wilson loops as topological indices: an example on the triangular lattice. Phys. Rev. B 99, 045140 (2019).

55. Alexandradinata, A., Dai, X. \& Bernevig, B. A. Wilson-loop characterization of inversion-symmetric topological insulators. Phys. Rev. B 89, 155114 (2014).

56. Hwang, Y., Ahn, J. \& Yang, B.-J. Fragile topology protected by inversion symmetry: diagnosis, bulk-boundary correspondence, and Wilson loop. Phys. Rev. B 100, 205126 (2019)
57. Song, Z.-D. et al. Delocalization transition of a disordered axion insulator. Phys Rev. Lett. 127, 016602 (2021)

58. Yang, Y. et al. Time-reversal-symmetry-broken quantum spin Hall effect. Phys. Rev. Lett. 107, 066602 (2011).

59. Li, H., Sheng, L. \& Xing, D. Y. Connection of edge states to bulk topological invariance in a quantum spin hall state. Phys. Rev. Lett. 108, 196806 (2012).

60. Alexandradinata, A., Dai, X. \& Bernevig, B. A. Wilson-loop characterization of inversion-symmetric topological insulators. Phys. Rev. B 89, 155114 (2014).

61. Chen, $C$. et al. Universal approach to magnetic second-order topological insulator. Phys. Rev. Lett. 125, 056402 (2020).

62. Kresse, G. \& Hafner, J. Ab initio molecular dynamics for liquid metals. Phys. Rev. $B$ 47, 558-561 (1993)

63. Kresse, G. \& Furthmüller, J. Efficient iterative schemes for ab initio total-energy calculations using a plane-wave basis set. Phys. Rev. B 54, 11169-11186 (1996).

64. Perdew, J. P., Burke, K. \& Ernzerhof, M. Generalized gradient approximation made simple. Phys. Rev. Lett. 77, 3865-3868 (1996).

65. Anisimov, V. I., Zaanen, J. \& Andersen, O. K. Band theory and mott insulators: Hubbard U instead of stoner I. Phys. Rev. B 44, 943-954 (1991).

66. Mostofi, A. A. et al. An updated version of wannier90: a tool for obtaining maximally-localised wannier functions. Comput. Phys. Commun. 185, 2309-2310 (2014).

\section{ACKNOWLEDGEMENTS}

The authors thank Wei Li for valuable discussion. This work was supported by the National Key Research and Development Program of China (2018YFA0307000), and the National Natural Science Foundation of China (11874022).

\section{AUTHOR CONTRIBUTIONS}

G.X. conceived the project. A.L. performed the first-principles calculations. Z.S. and A.L. did the symmetry analysis. All the authors contributed to the discussion and writing of the paper.

\section{COMPETING INTERESTS}

The authors declare no competing interests.

\section{ADDITIONAL INFORMATION}

Correspondence and requests for materials should be addressed to Gang $\mathrm{Xu}$.

Reprints and permission information is available at http://www.nature.com/ reprints

Publisher's note Springer Nature remains neutral with regard to jurisdictional claims in published maps and institutional affiliations.

\begin{abstract}
Open Access This article is licensed under a Creative Common Attribution 4.0 International License, which permits use, sharing, adaptation, distribution and reproduction in any medium or format, as long as you give appropriate credit to the original author(s) and the source, provide a link to the Creative Commons license, and indicate if changes were made. The images or other third party material in this article are included in the article's Creative Commons license, unless indicated otherwise in a credit line to the material. If material is not included in the article's Creative Commons license and your intended use is not permitted by statutory regulation or exceeds the permitted use, you will need to obtain permission directly from the copyright holder. To view a copy of this license, visit http://creativecommons. org/licenses/by/4.0/
\end{abstract}

(c) The Author(s) 2022 